\title{
Changes in Flood Risk under Global Warming Estimated Using MIROC5 and the Discharge Probability Index
}

\author{
Atsushi OKAZAKI \\ Institute of Industrial Science, University of Tokyo, Tokyo, Japan \\ Pat J. -F. YEH \\ International Centre for Water Hazard and Risk Management, Tsukuba, Japan \\ Kei YOSHIMURA \\ Institute of Industrial Science, University of Tokyo, Tokyo, Japan \\ Atmosphere and Ocean Research Institute, University of Tokyo, Kashiwa, Japan \\ Masahiro WATANABE, Masahide KIMOTO \\ Atmosphere and Ocean Research Institute, University of Tokyo, Kashiwa, Japan \\ and
}

Taikan OKI

Institute of Industrial Science, University of Tokyo, Tokyo, Japan

(Manuscript received 28 February 2011, in final form 9 May 2011)

\begin{abstract}
We evaluated change in flood risk under global warming using the output from the latest version of the Model for Interdisciplinary Research on Climate (MIROC5), an atmosphere-ocean general circulation model. River discharge for the $21^{\text {st }}$ century were simulated for the two Representative Concentration Pathway (RCP4.5 and RCP8.5) scenarios and converted to the Discharge Probability Index (DPI) to evaluate future flood risk. The occurrence of flood events corresponding to various DPI categories was calculated for each continental region. The results show a significant increase in the risk of massive flood incidents during the $21^{\text {st }}$ century in Asia, Africa, Oceania, and South America, with relatively large differences between the two scenarios. In contrast, both scenarios showed only slight increases in massive flood risk in North America and almost no change in Europe. For the RCP8.5 scenario in particular, the risk of massive flood occurrence will increase approximately ten times in Africa, seven times in Asia, and five times in South America by the end of the current century. Further analyses indicated that these projected flood increases will occur mainly due to the increases in the number of rainy days and the annual maximum daily precipitation, and the decrease in snowmelt in high latitudinal regions will play an important role on the unchanged risk in Europe in spite of the projected increase in precipitation.
\end{abstract}

Corresponding author: Atsushi Okazaki, Institute of Industrial Science, 4-6-1 Meguro, Komaba, Tokyo 1538505, Japan

E-mail: okazaki@rainbow.iis.u-tokyo.ac.jp

(C)2012, Meteorological Society of Japan

\section{Introduction}

Rivers are indispensable freshwater resources, but can also pose serious threats during flooding. According to the Fourth Assessment Report (AR4) of the Inter- 
governmental Panel on Climate Change (IPCC WG2 2007), increased flood occurrence can be anticipated in the $21^{\text {st }}$ century as the Earth's temperature continues to rise at the global scale. In 2011, for example, a massive flood in Thailand killed approximately 600 people and affected more than 5 million people, with overall economic loss exceeding $\$ 45.7$ billion (Thai Government 2011; The World Bank 2011). However, the Special Report on Managing the Risks of Extreme Events and Disasters to Advance Climate Change Adaptation (SREX 2011) noted that there is still low to medium confidence in projected future flood risk. Further study is therefore necessary.

Research on the potential impacts of global warming on water resources and flood drought disasters has increased during the past decade (Barnett et al. 2008; Labat et al. 2004; Matthews 2006; IPCC WG2 2007; SREX 2011). Numerous studies have suggested that global warming is likely to alter the hydrological cycle (Huntington 2006; Jung et al. 2010; Milly et al. 2005; Oki and Kanae 2006). Under conditions of rising temperature, precipitation is more likely to arrive in the form of heavy rains accompanied by an increase in flood risk (Allen and Ingram 2002; Goswami et al. 2006; Min et al. 2011, Trenberth 1998; Trenberth et al. 2003). Decreased accumulation and melting of seasonal snow packs may also cause a shift in the timing and amount of runoff and alter the availability of future water resources (Barnett et al. 2005, 2008).

Motivated by the IPCC, several studies have examined future flood risk under a warming climate. Nitta et al. (2010) estimated the future risk of floods in Japan using output data from a regional climate model provided by the Japan Meteorological Research Institute and concluded that massive floods will occur more frequently in Japan by the end of this century. At the global scale, however, only a few studies have examined future flooding. One example is the study of Hirabayashi et al. (2008) who projected future extreme river discharge under global warming using the older version of the Model for Interdisciplinary Research on Climate (MIROC3.2). Their results indicated increases in flood frequency over most regions of the world, except for North America and central to western Eurasia. In this study, we used the $21^{\text {st }}$ century river discharge data simulated by the MIROC5 [which will be used for the forthcoming IPCC Fifth Assessment (AR5) Report] to analyze future flood occurrences under warming scenarios for $21^{\text {st }}$ century climate.

\section{Datasets}

MIROC is an atmosphere-ocean general circu- lation model (AOGCM) developed jointly by the Atmosphere and Ocean Research Institute (AORI), University of Tokyo, the National Institute for Environmental Studies (NIES), and the Japan Agency for Marine-Earth Science and Technology (JAMSTEC). MIROC5 (Watanabe et al. 2010) is an improved version of the MIROC3.2 model used in the IPCC AR4 (2007). Although MIROC3.2 simulated mean precipitation similar to the multi-model average in the Coupled Model Intercomparison Project Phase 3 (CMIP3), it still had some shortcomings such as the exceedingly weak El Niño-Southern Oscillation (ENSO) simulation and unsatisfactory cloud representation. A MIROC4 version is also available, but although it has a finer grid resolution, the parameterization schemes for physical processes are not improved from the earlier MIROC3.2 version. Emori (2006) stressed that the prediction of climatic sensitivity depends more on the parameterization schemes of climate models than the model grid resolution. The parameterization schemes of cloud convection in MIROC5 have been significantly improved from those in MIROC3.2 (Watanabe et al. 2010). The dynamical cores of the atmosphere model and the radiation, cumulus convection, turbulence, and aerosol schemes have all been upgraded in MIROC5. For the ocean and land surface models in MIROC5, the sea ice component was improved, and an advanced version of the river routing model Total Runoff Integrating Pathways (Oki and Sud 1998) has been incorporated. The improved TRIP scheme (TRIP 2.0) uses a newly developed river network with T-85 resolution (Yamazaki et al. 2009) and an improved method to calculate river discharge (Ngo-Duc et al. 2007) in which variable flow velocity is applied to model short-term streamflow fluctuations. These features of MIROC5 have resulted in improved precipitation simulations, zonal-mean atmospheric fields, equatorial ocean subsurface fields, and the ENSO [Please see Watanabe et al. (2010) for more detailed information about MIROC5]. Therefore, we used MIROC5 because it can better simulate precipitation using the improved parameterization schemes. To enhance the reliability of the assessment of future flood risk, it is preferable to use multi-model ensembles of climate model simulations whenever available. However, because the latest simulation data prepared for AR5 were not yet fully available during our study and river discharge output is lacking from some climate models, we used only the data from the three ensembles of the MIROC5 simulations for each climate scenario.

MIROC5 data with T-85 grid resolution $(\sim 140 \mathrm{~km})$ were examined for the following three 20 -year peri- 
Table 1. Summary of observed and simulated river discharge in 15 global large river basins and the corresponding relative root-mean-square error (RRMS).

\begin{tabular}{llccc}
\hline River basin & \multicolumn{1}{c}{ Station } & Observation $\left(\mathrm{m}^{3} / \mathrm{s}\right)$ & Simulation $\left(\mathrm{m}^{3} / \mathrm{s}\right)$ & RRMS $(\%)$ \\
\hline Amazon & Obidos & 169987 & 111248 & 50.7 \\
Changjiang & Hankou & 22115 & 27741 & 50.5 \\
Columbia & Dalles & 5252 & 5294 & 66.7 \\
Congo & Kinshasa & 44931 & 82117 & 124.7 \\
Danube & Ceatal Izmail & 7894 & 5819 & 42.6 \\
Ganges & Farraka & 12085 & 16194 & 88.3 \\
Mekong & Stung Treng & 13239 & 11703 & 33.4 \\
Mississippi & Vicksburg & 18944 & 17861 & 21.3 \\
Murray & Lock 9 upstream & 295 & 2363 & 763.1 \\
Nile & El Ekhasa & 1276 & 40616 & 3390.4 \\
Ob & Salekharad & 12729 & 12686 & 135 \\
Parana & Timbues & 17328 & 34190 & 220.5 \\
Volga & Volgograd Power Plant & 8243 & 6798 & 94.6 \\
Yenisey & Igarka & 18964 & 12276 & 55.3 \\
Yukon & Pilat station & 6608 & 11022 & 177.1 \\
\hline
\end{tabular}

ods: 1980-1999, 2030-2049, and 2080-2099. The data from the 1980-1999 historical run were used for model evaluation against an observed hydrologic dataset, whereas those of the 2030-2049 and 2080-2099 runs were obtained to assess future flood risk under the RCP4.5 and RCP8.5 scenarios, respectively. The RCP4.5 scenario is based on a projection which assumes that radiative forcing stabilizes at less than + $4.5 \mathrm{~W} / \mathrm{m}^{2}$ by 2100 compared to the pre-industrial level. The RCP8.5 assumes that radiative forcing will reach + $8.5 \mathrm{~W} / \mathrm{m}^{2}$ compared to the pre-industrial level by 2100 . By analyzing the simulations for both scenarios, the impact of changes in radiative forcing on future climate and the associated flood risk can be evaluated. For both scenarios, three ensemble simulations were used to determine future flood risk.

To evaluate MIROC5 simulations of precipitation and river discharge, observation data provided by the Global Precipitation Climatology Project (Huffman et al. 2009) and the Global Runoff Data Centre (GRDC: http://www.bafg.de/grdc.htm) were used. The GPCP global dataset has a 2.5-degree grid resolution. To allow for comparison with the MIROC5 output, the GPCP data were made compatible with T- 85 by bilinear interpolation. For the river discharge validation, 15 large river basins were selected globally (Table 1) covering most of the continents and global climatic zones, including tropical, arid, mid-latitude rainy, Asian monsoon, and high latitudes. The basins are shown in Fig. 1. Note that the observed river discharge data do not necessarily represent natural discharge because some rivers have been influenced by human activity, such as irrigation, diversions, and dam construction. Furthermore, because not all the basins had a complete GRDC data set for the period of 1980-1999, the climatology of available discharge data was used to compare with the MIROC5 data on a monthly scale.

\section{Model Validation}

\subsection{Precipitation}

The global mean annual precipitation agreeement between the MIROC5 simulation and GPCP precipitation data between 1980 and 1999 was evaluated by the root-mean-square error (RMSE) and the pattern correlation coefficient $(\mathrm{R})$. Global average precipitation was $2.67 \mathrm{~mm} /$ day from the GPCP data and $3.20 \mathrm{~mm} /$ day from MIROC5. Meanwhile the values produced by the high-resolution (hires) and medium-resolution (medres) versions of MIROC3.2 were $3.10 \mathrm{~mm} /$ day and $2.83 \mathrm{~mm} /$ day, respectively. The global average RMSE for monthly precipitation compared with GPCP was $1.25 \mathrm{~mm} /$ day, which represents an improvement over the RMSE of $1.50 \mathrm{~mm} /$ day, which was derived as the multi-model average in the CMIP3 Project (ranging from 1.10-1.99 $\mathrm{mm} /$ day, see Nohara et al. 2006). The global land-only RMSE was $1.42 \mathrm{~mm} /$ day. MIROC3.2 (hires) and MIROC3.2 (medres) produced values of $1.30 \mathrm{~mm} /$ day and $1.17 \mathrm{~mm} /$ day respectively. The $\mathrm{R}$ value of 0.86 is better than any of the global values obtained by the 19 GCMs used by Nohara et al. (2006, Table 2). The global land-only $\mathrm{R}$ values were $0.76,0.71$, and 0.68 by MIROC5, MIROC3.2 (hires), and MIROC3.2 (medres), respectively. Figure 2 shows (a) the difference in global pattern 1980-1999 mean 


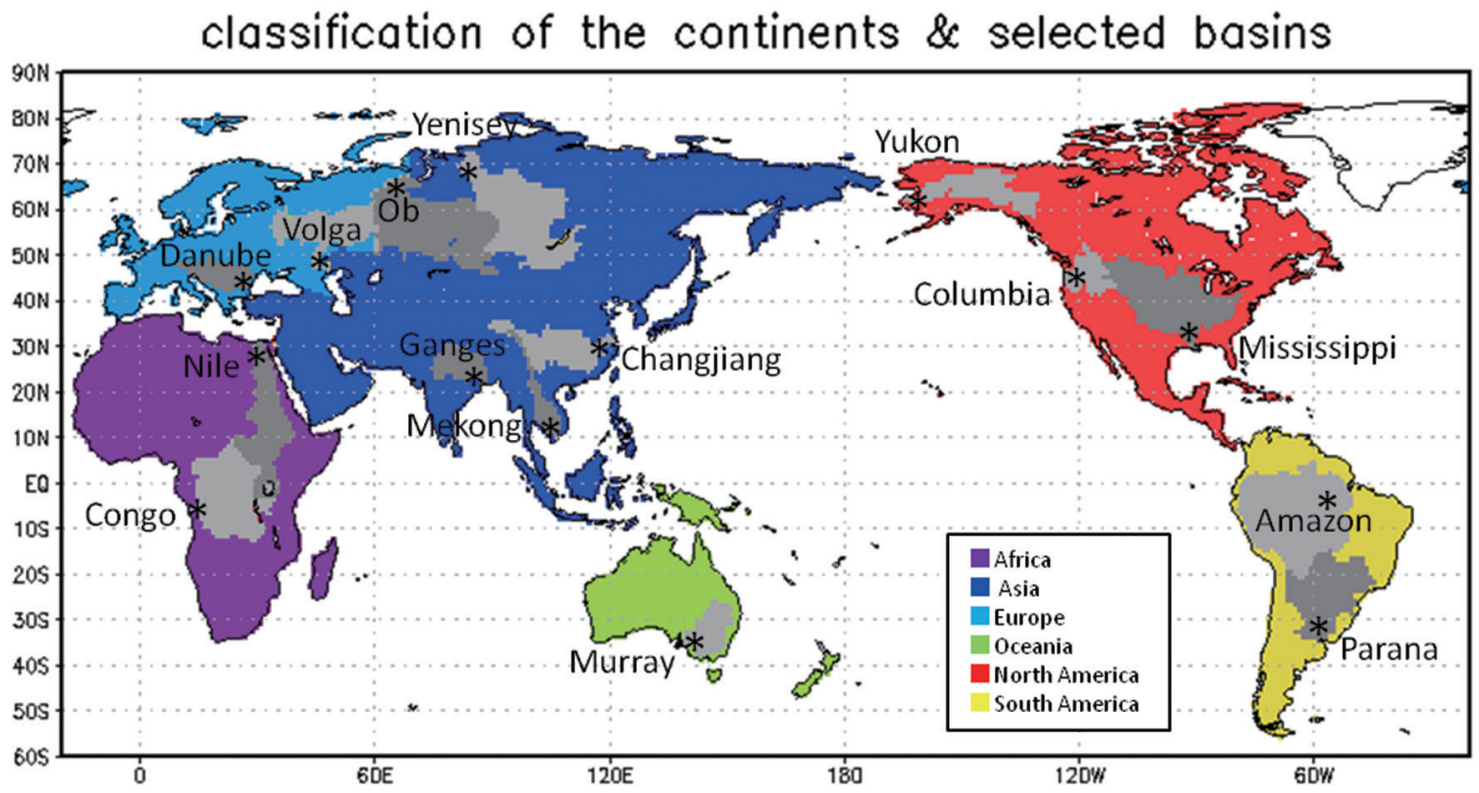

Fig. 1. Classification map of the selected 15 major river basins and continents. The Ob basin belongs to Asia and Volga belongs to Europe. Asterisks indicate the location of the discharge observation.

precipitation between the MIROC5 and GPCP values and (b) the corresponding global zonal mean precipitation comparison. Values produced by MIROC3.2 (hires) and MIROC3.2 (medres) are also shown in Fig. $2 b$ by dotted and short dashed lines, respectively. These results indicate that MIROC5 overestimated precipitation in central and southern Africa, most of Asia, western and central North America, and western South America. In contrast, MIROC5 underestimated precipitation in the north and west of Europe, southern Asia, and most of the Mississippi and Amazon river basins. Overall the reproducibility of the MIROC5 precipitation is fair to good with the exception of locations close to the equator (north and south of 15 degrees) where large biases can be noted. In addition, compared to MIROC3.2, MIROC5 showed similar or slightly poorer reproducibility of absolute values for the mean state of precipitation. As mentioned by Watanabe et al. (2010), the hydrological cycle simulation appears to be too active in MIROC5. However, spatial variability was better simulated by MIROC5 than by MIROC3.2. Also it should be worthwhile to note that GPCP may include some errors particularly at high-latitudes over land (Adler et al. 2011).

\subsection{River discharge}

The mean seasonal cycles of simulated 1980-1999 river discharges over the selected 15 large river basins are compared with the corresponding GRDC observations in Fig. 3, along with all three ensemble simulations. Discharges were well simulated for the Changjiang and Yenisey River basins. Discharges of the Mekong and Mississippi also appeared to be well simulated, but this may be because biases were cancelled out by the spatial average over a whole basin, Fig. 2a. Although the simulated peak discharges for the Ganges and Volga basins did not match local observations, the timing of the seasonal cycle of discharge was accurately reproduced. For the Amazon, Columbia, Danube, Ob, and Yukon river basins, however, the timing of simulated peak discharge was shifted forward by one or two months. In addition, the simulated discharge in the Congo, Murray, Nile, and Parana basins showed relatively low reproducibility. Comparison of simulated precipitation with GPCP data (not shown) indicated that the seasonal cycle of precipitation was generally well simulated by MIROC5, and the precipitation simulation had a better quality in general than the corresponding discharge simulation (particularly for the Parana and Murray basins). This suggests that factors other than precipitation (e.g., human activities of water regulation and evaporation from river surface in arid basins) may be the main contributors to the simulation biases of river discharge. 




Fig. 2. Climatology of annual mean precipitation ( $\mathrm{mm} /$ day). (a) Difference between simulated mean precipitation and observed GPCP data. (b) Zonal-mean precipitation simulated by MIROC5 (red) and two versions of MIROC3 (black) simulations and from the GPCP observations (blue).

Figure 4 plots the coefficient of variance $(\mathrm{CV})$ of observed and simulated monthly river discharge (1980-1999). CV is defined as the ratio of the standard deviation to the mean, indicating the model's ability to reproduce the observed interannual variability of streamflow. The $\mathrm{CV}$ of simulated discharge agreed well with that produced by the GRDC in the Changjiang, Ganges, Mekong, Yenisey, and Yukon basins, whereas the agreement was relatively poor in the Murray, Nile, and Parana basins.

Table 1 summarizes the observed and simulated mean river discharge for the 15 river basins and the corresponding relative RMSE (RRMS) between the simulated discharge and observation. The RRMS indicates the extent to which the simulated river discharge deviates from the observed value and is an index of reproducibility, as used by Nohara et al. (2006, Eq. 5). The magnitude of RRMS in Table 1 is very close to that of the multi-model average presented by Nohara et al. (2006, Table 4). Considering that it was not constrained by observed precipitation, the quality of the MIROC5 simulated global hydrology in this study was considered acceptable for the purpose of evaluating future hydrologic changes following global warming.

\section{Projection of $21^{\text {st }}$ century Precipitation and River Discharge}

\subsection{Precipitation and evapotranspiration}

Figure 5 presents the global percentage change in future precipitation (i.e., 2080-2099 mean precipitation minus 1980-1999 mean precipitation and then divided by the latter) from the MIROC5 simulations of the RCP4.5 (left) and RCP8.5 (right) scenarios. Only global regions with a statistically significant change $(>90 \%)$ over two different periods based on a $\mathrm{t}$-test are colored in the figure. The projections indicate an increase in mean precipitation for 2080-2099 for almost all the Northern Hemisphere land area, including eastern Africa, continental Eurasia (except central and southern Europe), Oceania, and the northern part of North America. The most significant precipitation increase was predicted for high-latitude areas. Few significant changes were shown for the Southern Hemisphere, although some reduction in precipitation was indicated in the southwestern US, southern South America, and southern Africa. The change in precipitation under the RCP8.5 scenario was generally larger than for RCP4.5. The pattern of mean precipitation change in 2030-2049 relative to 1980-1999 was similar to that shown in Fig. 5 but with a smaller magnitude (not shown). The simulated change in the future precipitation pattern was consistent with that summa- 

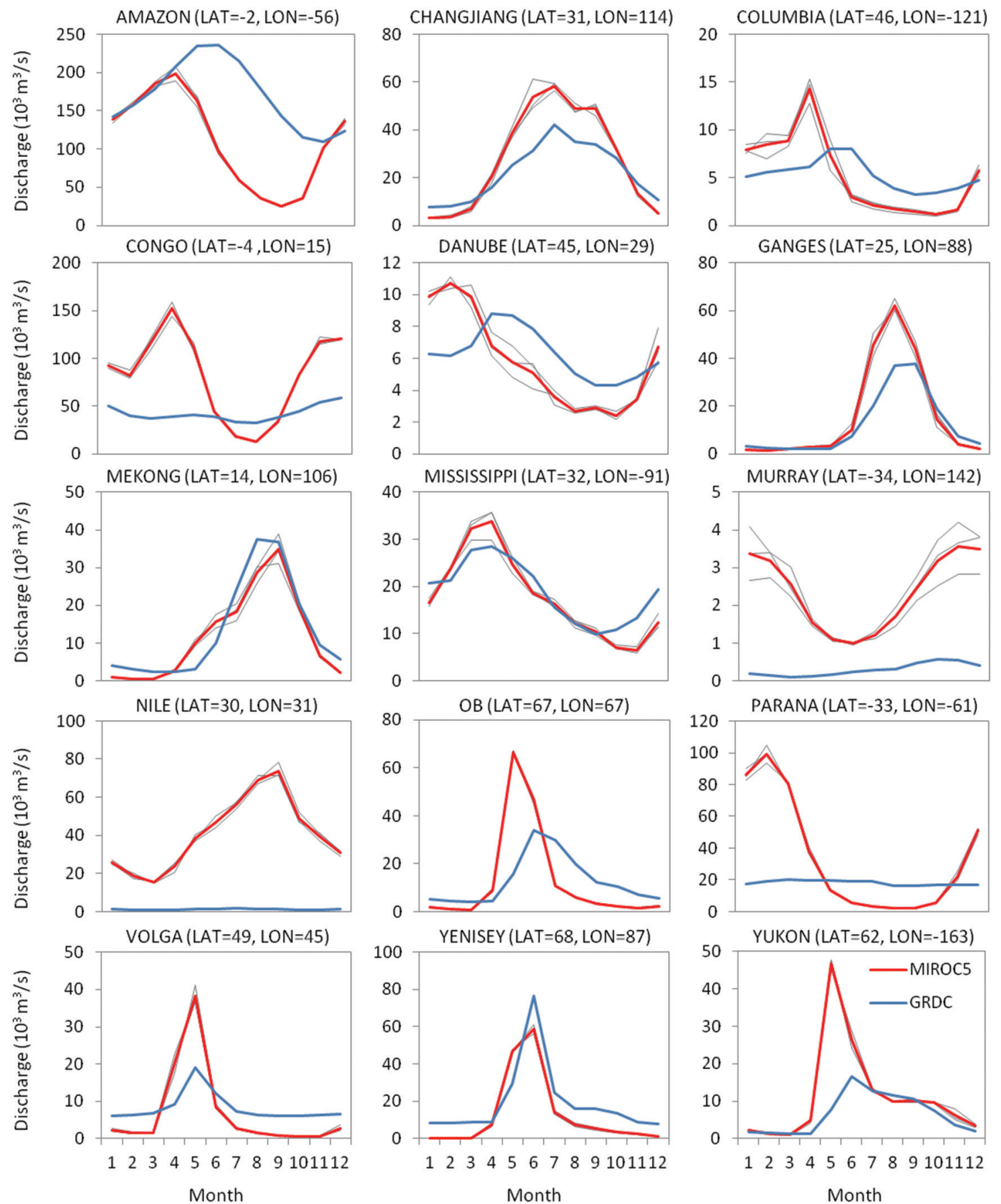

Fig. 3. Comparison of simulated (red) and observed (blue) long-term 1980-1999 average monthly river discharges for the selected 15 large river basins $\left(10^{3} \mathrm{~m}^{3} / \mathrm{s}\right)$. Simulations include three ensembles plotted in gray and the ensemble average in red. 

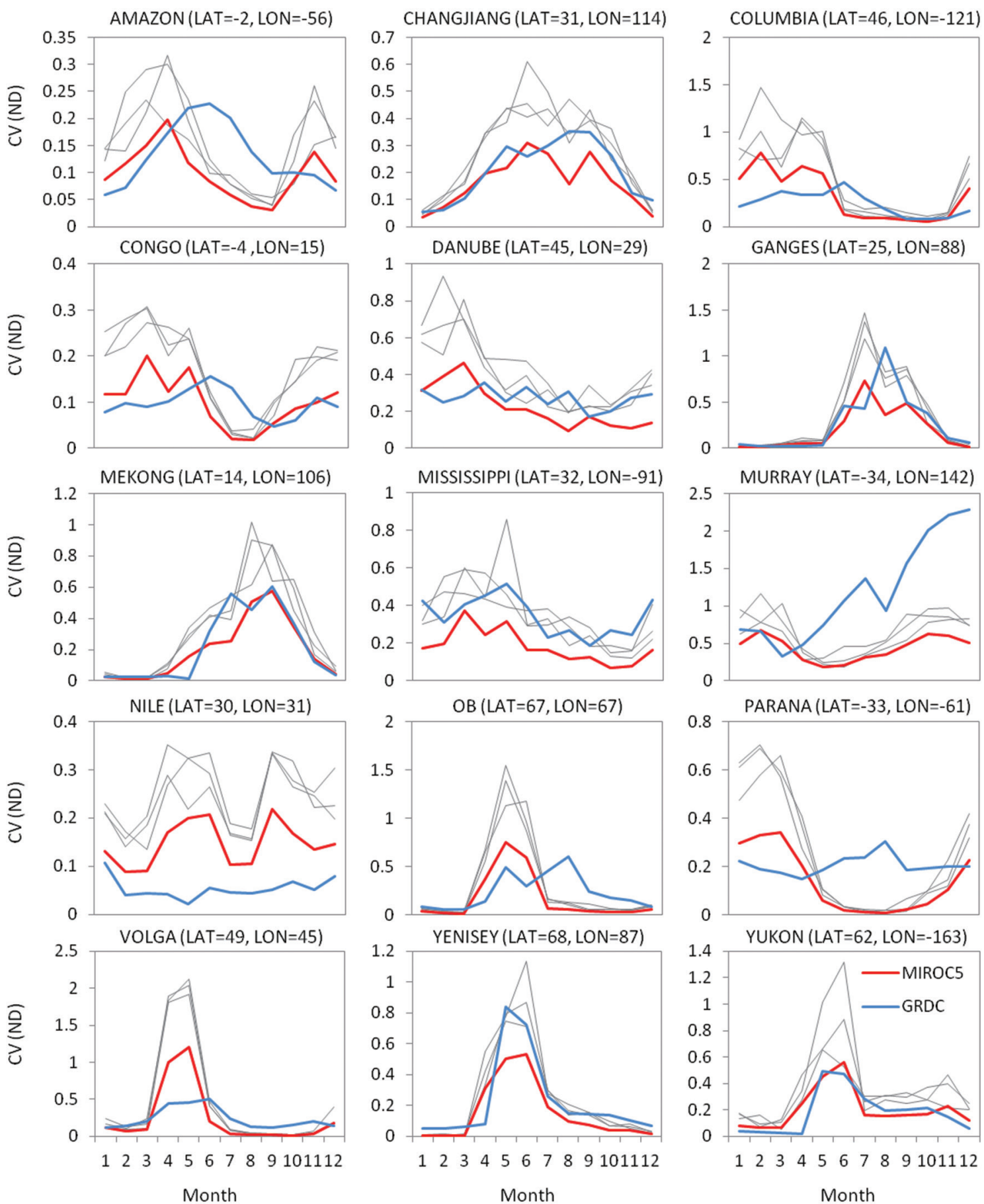

Fig. 4. Comparison of the coefficient of variance (CV) of simulated (red) and observed (blue) long-term (1980-1999) river discharges for the selected 15 large river basins (non-dimensional). Simulations include three ensembles plotted in gray and the ensemble average in red. 


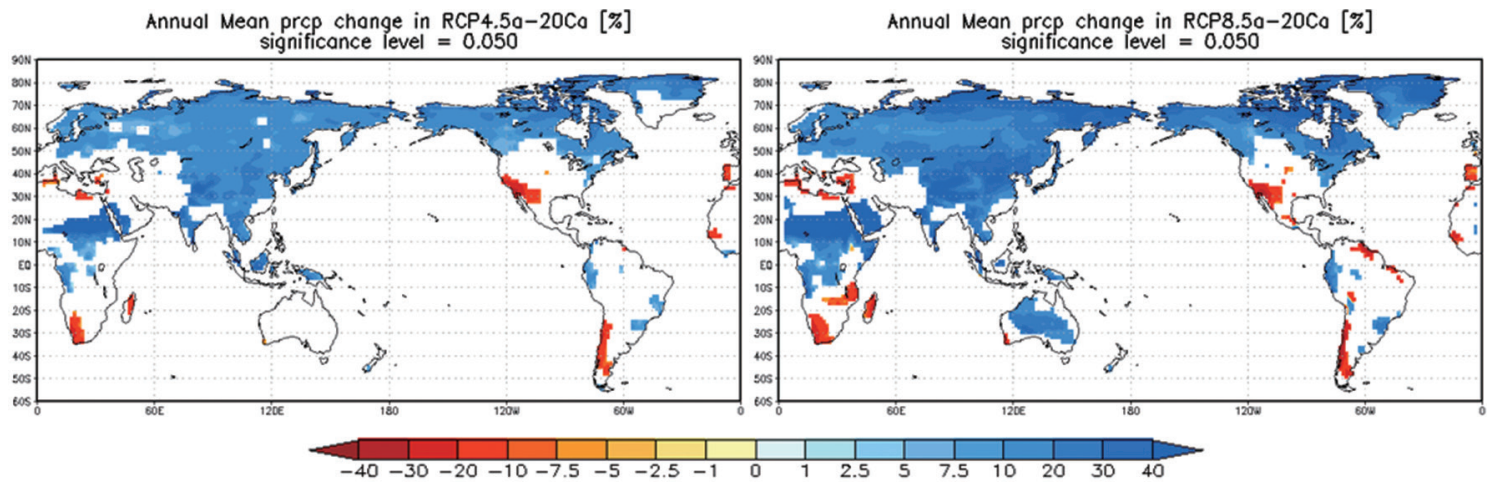

Fig. 5. Percentage change in future (2080-2099) average precipitation relative to the 1980-1999 average simulated by MIROC5 under both RCP4.5 (left) and RCP8.5 (right) scenarios (\%).

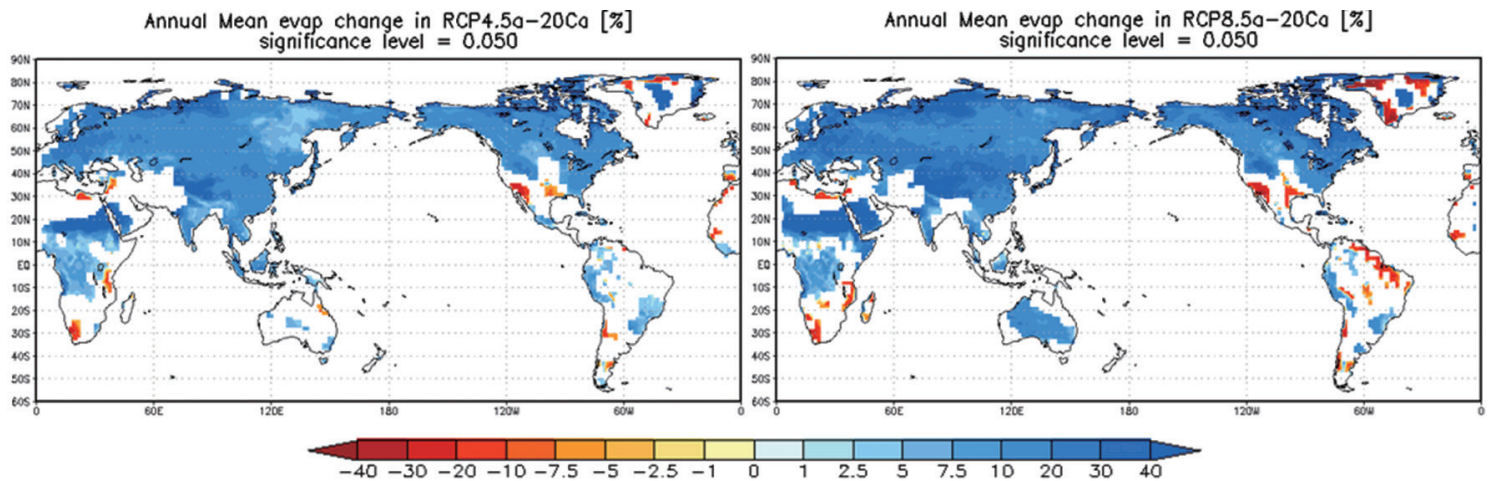

Fig. 6. Same as Fig. 5, but for evapotranspiration (\%).

rized by the AR4 (IPCC WG1 2007): precipitation will increase in high latitudes, whereas mid-latitude areas will generally be drier in the future.

Figure 6 illustrates that the change in predicted evapotranspiration was similar to the results for precipitation discussed above. The distributions of predicted changes are very similar to those predicted for precipitation. This indicates that the limiting factor for evapotranspiration is water availability, so that evapotranspiration increases (decreases) where precipitation increases (decreases). However, as described in the next section, the rate of increase or decrease is not the same as the rate of change in precipitation. Thus some distinctive differences in runoff become apparent.

\subsection{River discharge}

Figure 7 presents the percentage change of future (2080-2099 mean) river discharge relative to the $20^{\text {th }}$ century (1980-1999 mean) from the MIROC5 simulations for both the RCP4.5 (left) and RCP8.5 (right) scenarios. As in Fig. 5, only the regions with statistically significant changes are colored. The figure shows predicted decreases in future river discharge in central North America, southern South America, and most of Europe. In contrast, future river discharge in most of Asia, Oceania, and high-latitude areas of North America was predicted to increase, in a manner generally consistent with the projected change in precipitation (Fig. 5). However, in Europe, projections showed decreases in river discharge despite expected increases in future precipitation because the increase in future evaporation exceeded the relative increase in future precipitation (not shown).

\section{Evaluation of Future Flood Risk}

According to the MIROC5 simulations presented above, future river discharge will increase compared to the $20^{\text {th }}$ century (1980-1999) in regions such as Asia and high-latitude regions of North America. According to the AR4 (IPCC WG1 2007), continuing global 


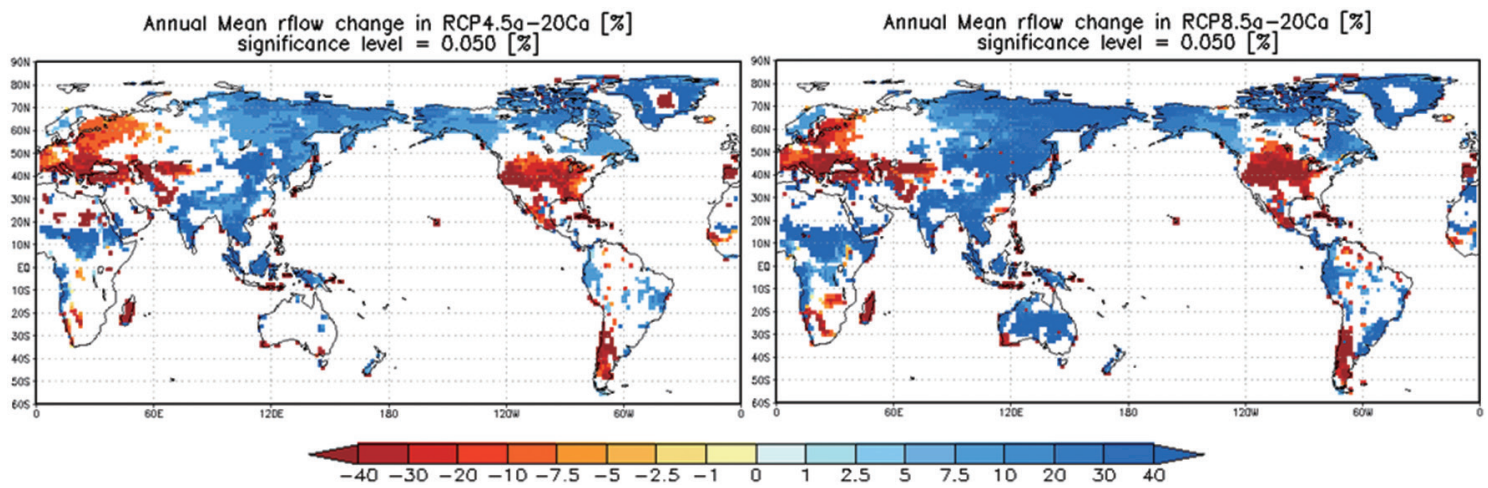

Fig. 7. Same as Fig. 5, but for river discharge (\%).

temperature rises will alter the global hydrologic cycle. Precipitation is more likely to arrive in the form of heavy rains accompanied by larger amounts of atmospheric water vapor as dictated by the Clausius-Clapeyron relationship (Trenberth et al. 2003). Therefore, the changes in frequency and magnitude of future floods are expected to be more severe than the changes in annual mean of future precipitation and river discharge. Moreover, the extent and timing of extreme floods are more critical than the mean river discharge from the perspective of disaster prevention and water resources management. It is therefore imperative to quantify likely changes in future extreme flood statistics. Figure 8 compares the observed and simulated daily discharge duration curves (i.e., sorted daily discharge of one year in decreasing order) for some of the selected large river basins in Table 1, as the daily discharge cannot be directly compared between the climate simulations and the actual observations. As shown in Fig. 8, the maximum daily discharge was well simulated in the Mekong and Yenisey basins. However, for other basins shown in this figure, the simulated daily discharge is not as well matched with the corresponding daily observations. For these rivers, there was a tendency for exponentially higher discharge in both the simulations and the observations. Therefore, the MIROC5 simulated river discharge data were converted into discharge probability index (DPI) values (Yoshimura et al. 2008) to evaluate future flood risk. The DPI represents the return period of flood occurrence calculated by estimating a probability distribution for the extreme values of river discharge, which can be used to directly assess flood risk. Yoshimura et al. (2008) validated the DPI with model-simulated river discharge during a typhoon event in Japan in 2004. The result showed that a high DPI calculated from the model output cor- responded well to actual flood damage records.

In estimating future flood risk using a simulated river discharge, there are at least two main issues to note. The first is the accuracy of future projection by the model, and the second is the difference between historical simulation and observation judged from the available historical data. Regarding the first issue, for which the true answer is unknown, the validation of MIROC5 simulations of precipitation and discharge against observed data (Figs. 2, 3) provided reasonable confidence in the projection of future river discharges. To overcome the second issue, the statistical approach of the DPI proposed by Yoshimura et al. (2008) was used here. The concept of the DPI (with units of years) is that the probability of exceeding a threshold discharge $D(\Pi)$ in a given year is $1 / \Pi$ and the expected occurrence is once in $\Pi$ years. To calculate the threshold discharge $D(\Pi)$, the Poisson distribution and an exponential distribution are assumed to represent the interval and magnitude of peak discharge that exceed a specific threshold value, respectively. Following this theory (Hoshi 1998), a Gumbel distribution is introduced as the probability distribution of annual maximum discharge. Therefore, the $D(\Pi)$ can be calculated as

$$
D(\Pi)=\mu+\beta \ln \left(-\ln \left(1-\frac{1}{n}\right)\right)
$$

with $\beta$ (a scale parameter) and $\mu$ (a location parameter) given as follows:

$$
\begin{aligned}
& \beta=\frac{1}{M} \sum_{i=1}^{M}\left(D_{i}-D_{M}\right) \\
& \mu=D_{M}+\beta \ln \lambda \\
& \lambda=\frac{M}{N}
\end{aligned}
$$



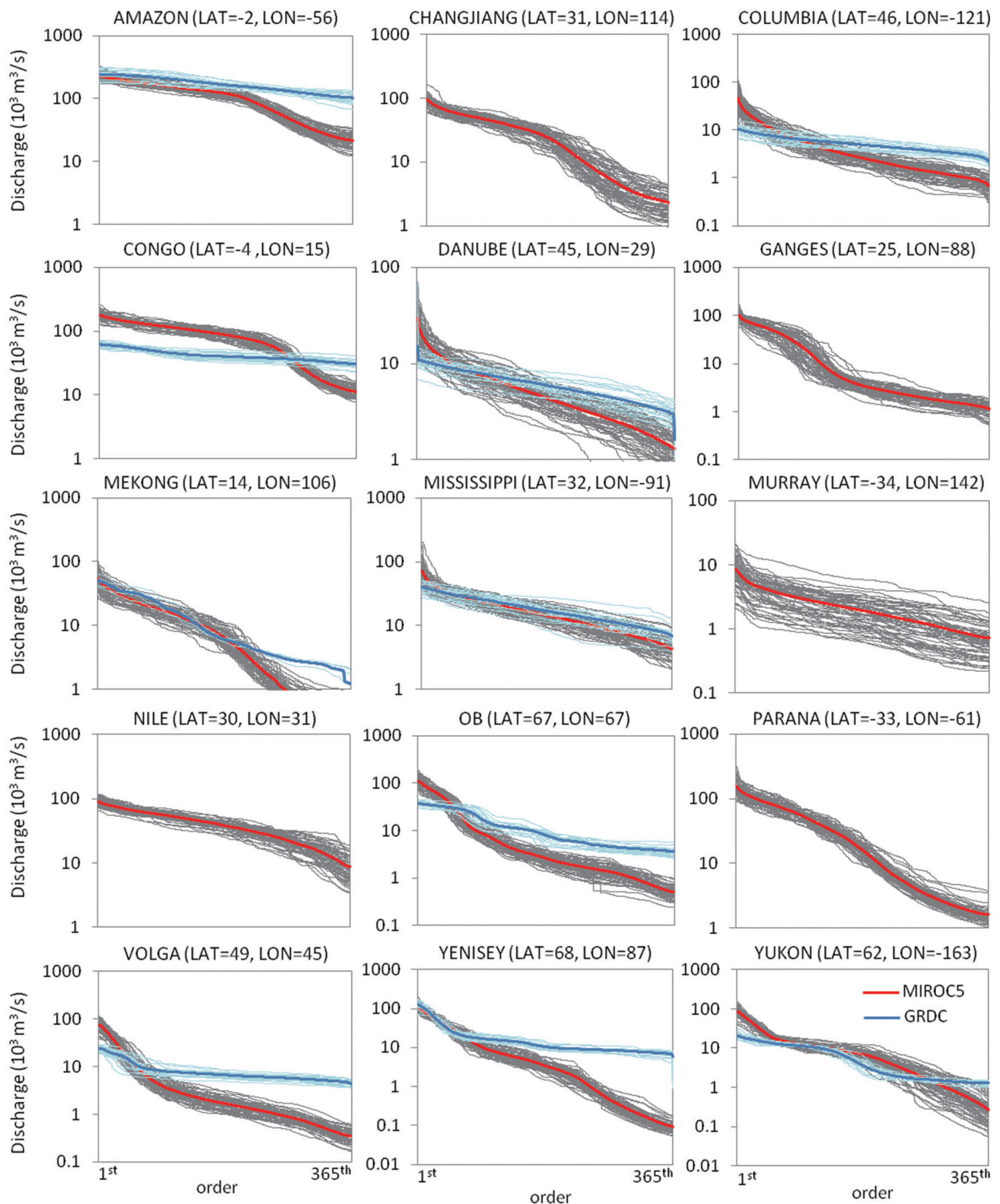

Fig. 8. The flow duration curve based on the 1980-1999 daily river discharge data. The red line is based on the ensemble average of the MIROC5 simulated river discharge $\left(10^{3} \mathrm{~m}^{3} / \mathrm{s}\right)$. The blue line is based on the GRDC observation, only where data are available $\left(10^{3} \mathrm{~m}^{3} / \mathrm{s}\right)$. 
where $D_{i}$ indicates the $i^{\text {th }}$ maximum, $D_{M}$ is the threshold discharge, $N$ is the number of years $(=20$ in this study), $M$ is the number of samples, and $\lambda$ is a constant of the frequency of annual occurrence. The optimal value for $\lambda$ is likely to be different for each grid, but it is difficult to estimate in practice because of limited data availability. Instead of estimating $\lambda$ for each grid, a sensitivity test was undertaken which indicated that the number of flood occurrences was relatively insensitive to the $\lambda$ value (not shown). This finding indicated that the model's upper discharge predictions fit to an exponential function that we assumed to be independent of the value for $\lambda$. Therefore, in this study a constant of $\lambda=$ 3 was adopted following the value used by Yoshimura et al. (2008). Thus, $\mathrm{M}=60$ and $D_{M}$ denotes the $60^{\text {th }}$ maximum daily discharge during the 1980-1999 period. The first 60 largest daily discharges during 1980-1999 from the simulation were used to calculate $D(\Pi)$, and then the number of floods exceeding $D(\Pi)$ in each period $(1980-1999,2030-2049$, and 2080-2099) were counted. For the two future periods, both scenarios were considered. Finally, the spatial average was obtained over each continent according to the classification map shown in Fig. 1 to provide an overview of projected changes in flooding, as only a few studies on a continental or global scale have previously been undertaken.

Figure 9 presents the calculated flood occurrence, with each bar graph representing the number of days of flood occurrence for different time periods and scenarios. Because of the large differences in numbers of flood occurrences, three categories of DPI are plotted separately: DPI $=1-10$ years (small flood), 10-100 years (medium flood), and over 100 years (massive flood) in Fig. 9. One, two, and three asterisks indicate significance at the $90 \%, 95 \%$, and $99 \%$ significance levels, respectively. The error bars indicate the standard deviation of the flood occurrence days based on three ensembles of MIROC5 simulations. These results show significant increases in future flood risk in all three flood-level categories in Asia, Africa, Oceania, and South America, where the differences between the RCP4.5 and RCP8.5 scenarios were large. In contrast, a slight increase in future massive flood risk was found in North America, whereas no significant change was found in Europe under both scenarios.

For Africa, the risk of a massive flood occurrence was projected to increase by nearly tenfold in 2080 2099 under the RCP8.5 scenario and by about four times for other combinations of time period and scenario. For medium and small floods, the future risk is increased by approximately one to four times among the different cases in Africa. For Asia, both massive and medium floods were expected to increase in occurrence by approximately four times during the 2080 2099 period under RCP8.5, and by about twice for other cases. In Oceania, an approximately three-fold increase in massive and medium floods was projected for 2080-2099 in the RCP8.5 scenario, with a much smaller increase expected for other cases. For South America, massive and medium floods were predicted to increase by approximately five times during the 2080-2099 period (RCP8.5), with only slight increases for other cases.

For Europe and North America, less change in flood risk was identified for the $21^{\text {st }}$ century. For Europe, small floods were predicted to decrease by $\sim 30 \%$ in 2080-2099 under both scenarios, and almost no change in risk was identified for medium to massive floods. For North America, the number of small and medium flood occurrences was predicted to remain almost the same as at present under both scenarios, but massive floods will become nearly twice as frequent during 2080-2099 (RCP8.5).

Hirabayashi et al. (2008) analyzed future flood risk by only the grids in which annual maximum discharge (flood magnitude) will increase significantly in the $21^{\text {st }}$ century. However, there are some regions where the maximum discharge does not change considerably, but the number of flood occurrences does increase significantly. To investigate both flood frequency and magnitude, a t-test was conducted on both the number of annual flood occurrences and the annual maximum discharge. The result, based on both the RCP 4.5 and RCP 8.5 simulations, is shown in Fig. 10 where the global regions with statistically significant changes are highlighted. As shown in this figure, the global regions that will experience increased flood occurrence almost overlap with the regions with a more significant future flood magnitude, indicating that floods in the $21^{\text {st }}$ century are likely to be more disastrous than those at present. Conversely, the regions where future flood occurrences will decrease such as northern North America and East Europe do not match well with the regions with a reduced flood magnitude, suggesting that in these regions the future flood risk will probably remain the same as the present. Therefore, the overall global flood risk is expected to increase under a future warming climate.

Another important aspect to consider is the cause of the changes in future flood risk. Figure 11 shows the global regions with statistically significant changes in 2080-2099 based on the RCP scenarios; this figure is similar to Fig. 10, but depicts the number of rainy days 
Significance level: * $90 \%, * * 95 \%, * * * 99 \%$
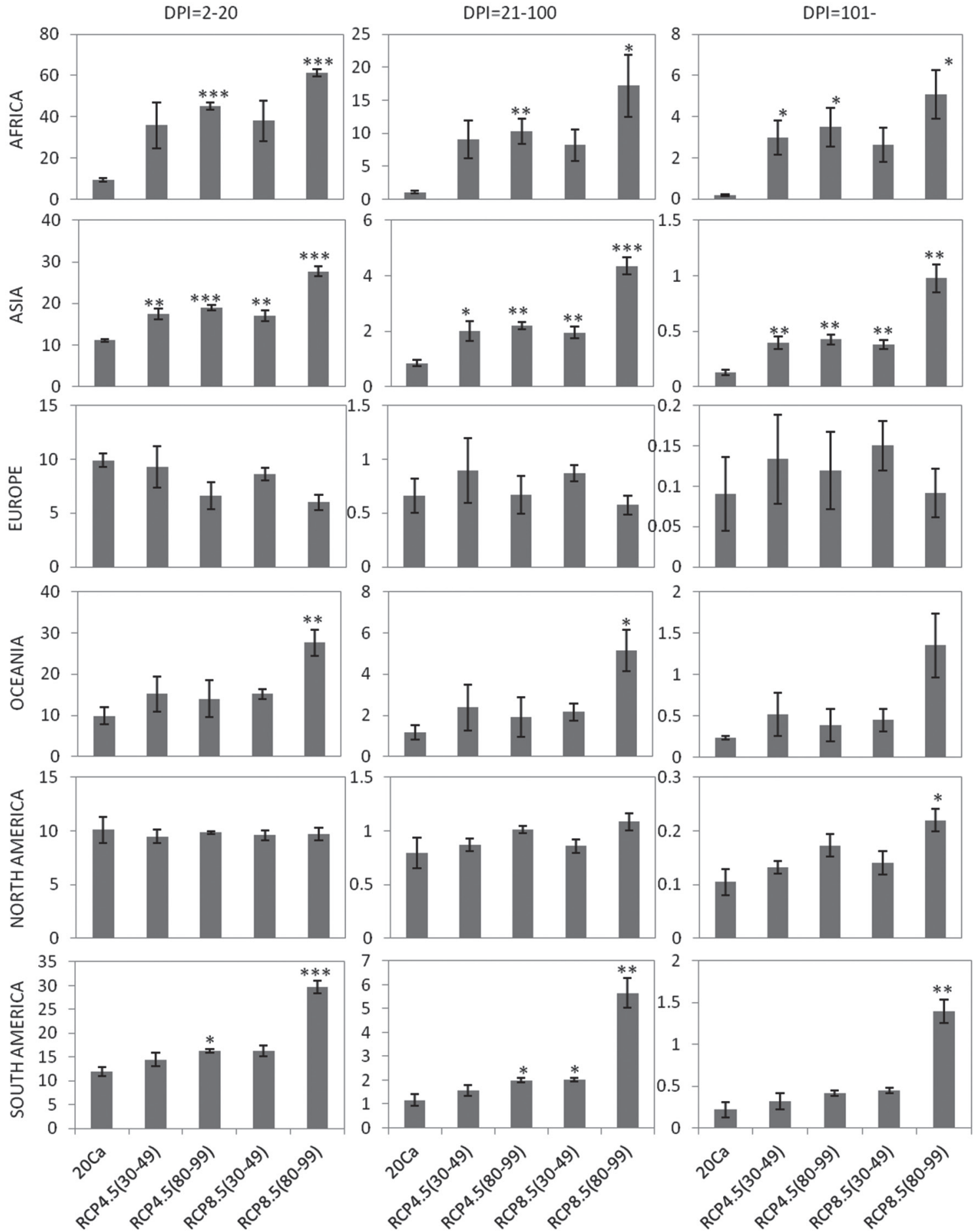

Fig. 9. The number of days of flood occurrence in each continent for the 1980-1999, 2030-2049, and 2080-2099 periods under the RCP4.5 and RCP8.5 scenarios, corresponding to three DPI categories (small, medium, and massive floods). One, two, and three asterisks indicate $90 \%, 95 \%$, and $99 \%$ levels of significance, respectively. 


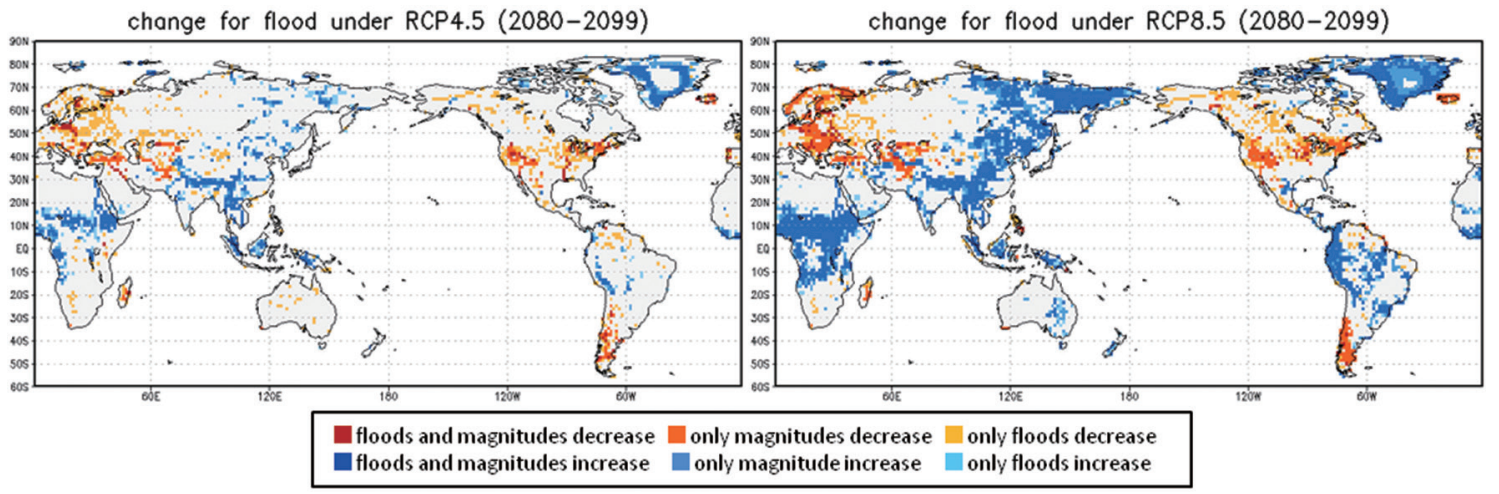

Fig. 10. Global maps showing statistically significant change in the number of flood occurrences and the annual daily maximum discharge. (The significance level is $90 \%$. Blue indicates a statistically significant increase and red indicates a significant decrease).
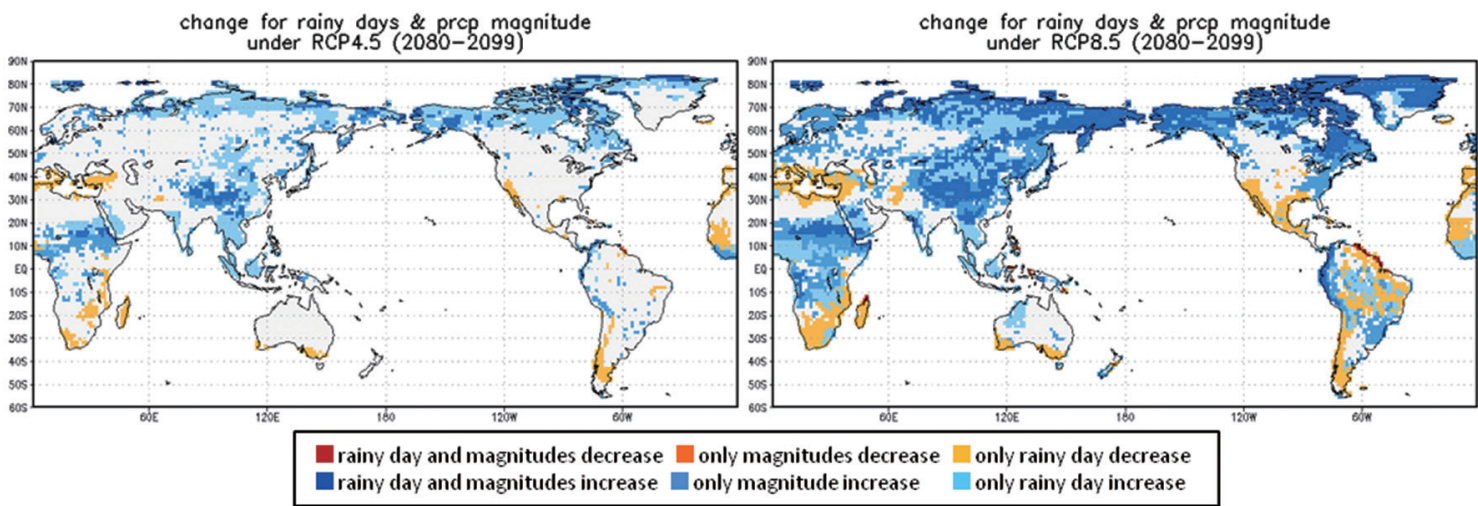

Fig. 11. Similar to Fig. 10, but for the number of rainy days and the annual maximum daily precipitation.

and the annual maximum daily precipitation. The figure clearly shows that the increase in flood frequency and magnitude (Fig. 10) is directly linked to the increase in the numbers of rainy days and the precipitation amount, as Kundzewicz et al. (2008) and Bates et al. (2008) argued. However, this figure also shows some high-latitude areas (in northern North America and northern Europe) with predicted increases in precipitation frequency and magnitude, but predicted decreases in flood frequency and magnitude. In both areas, floods are mainly caused by snowmelt as indicated by Barnett et al. (2005). Figure 12 shows the global regions with statistically significant changes in annual maximum snowmelt on five consequent days during the 2080 2099 period based on the RCP scenarios as with Fig. 10. The area with a predicted decrease in snowmelt in Fig. 12 matches the high-latitude areas mentioned above. In these areas, the earlier shifts of spring peak flows are projected by MIROC5, as found by Hirabayashi et al. (2008); however, such shifts do not affect flood occurrence and magnitude since a decrease in snowfall is also projected in the affected areas.

These results are qualitatively comparable with those of Hirabayashi et al. (2008), who used MIROC3.2 with the SRES A1B scenario. The global locations where flooding will increase or decrease are almost identical to the regions of increased flood risk in most of monsoon Asia, tropical Africa, and tropical South America and decreased flood risk in eastern and northern Europe and most parts of northern North America, respectively. As a whole, larger areas are threatened by floods in the MIROC3.2 prediction than in MIROC5. However, differences can be seen in western and southern Europe except for the Iberian Peninsula and coastal areas of 
chonge for 5-days snowmelt under RCP4.5 (2080-2099)

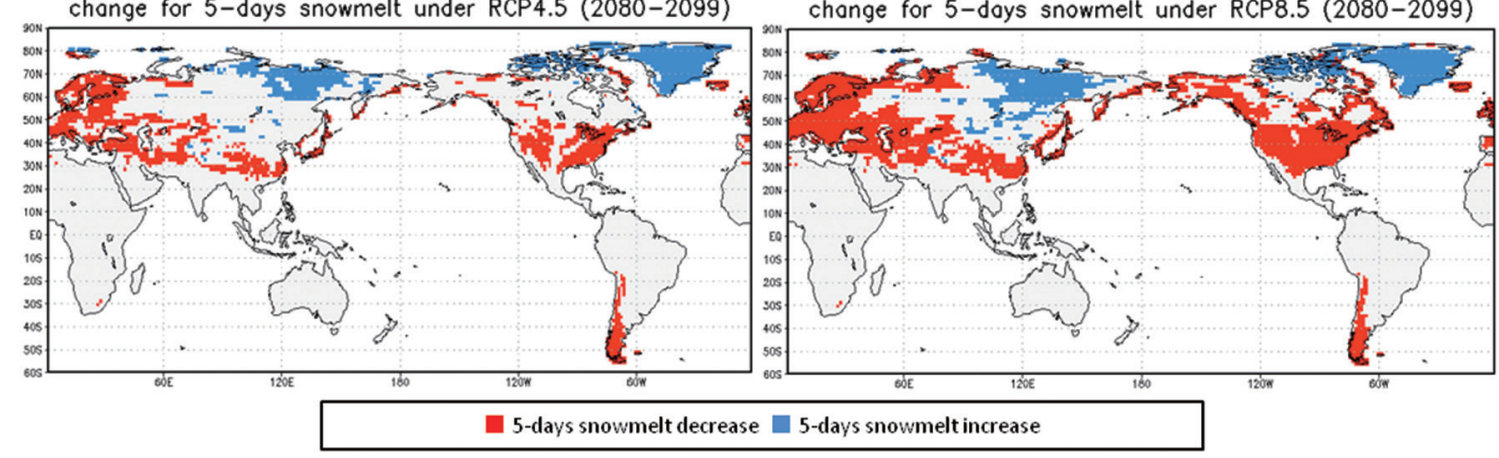

Fig. 12. Similar to Fig. 10, but for the annual maximum snowmelt on five consequent days. the United States. These differences are attributable to the different scenarios used in future simulations and also to the model's overall performance. The similarity of overall trends should still be stressed, even though many model aspects have been changed from the previous version of MIROC3.2. Overall, the results indicate added reliability in the newer version.

\section{Conclusions}

We analyzed changes in future flood risk for each continent under global warming conditions by simulating the $21^{\text {st }}$ century using the AOGCM MIROC5. Two scenarios, RCP4.5 and RCP8.5, were examined in three 20-year study periods: 1980-1999, 2030-2049, and 2080-2099. For each period and each continent, the average numbers of flood occurrences corresponding to different DPI categories were calculated.

For massive flood occurrences, a significant increase in future flood risk is expected for Asia, Africa, Oceania, and the South American continent, where large differences were found between the RCP4.5 and RCP8.5 scenarios. In contrast, a slight increase of massive flood risk in North America, and almost no change in Europe are anticipated under both scenarios. In particular, if the radiative forcing reaches $+8.5 \mathrm{~W} / \mathrm{m}^{2}$ compared to the pre-industrial level by 2100 (scenario RCP8.5), the risk of massive flood occurrence will increase approximately ten times in Africa, seven times in Asia, and five times in South America by the end of the $21^{\text {st }}$ century (2080-2099). Further analyses indicated that these projected flood increases will occur mainly due to the number of rainy days and the annual maximum daily precipitation, and the decrease in snowmelt in high latitudinal regions will play an important role on the unchanged risk in Europe in spite of the projected increase in precipitation.
Only MIROC5 was used in this study, and we emphasize the need for further study using multi-model ensemble data. Moreover, representations of land-surface processes in climate models must continue to be improved, particularly to incorporate human impacts on global hydrology. Such improvements are critical for obtaining more reliable projections of future $\left(21^{\text {st }}\right.$ century) global water resources and flood occurrence and will be priorities for future research.

\section{Acknowledgments}

This work was supported by the Japanese Ministry of Education, Culture, Sports, Science, and Technology through the Innovative Program of Climate Change Projection for the $21^{\text {st }}$ Century (the Kakushin Project), JSPS KAKENHI, Grants-in-Aid for Scientific Research (S) (23226012), and Global Environment Research Fund (GERF; S8) from the Ministry of Environment. Furthermore, the first author is financially supported by Global Center of Excellence for Sustainable Urban Regeneration in the University of Tokyo for his PhD study.

\section{References}

Adler, R. F., G. Gu, and G. J. Huffman, 2011: Estimating Climatological Bias Errors for the Global Precipitation Climatology Project (GPCP), J. Appl. Meteor. Climatol., 51, 84-99.

Allen, M. R., and W. J. Ingram, 2002: Constraints on the future changes in climate and the hydrological cycle, Nature, 419, 224-232, doi:10.1038/nature01092.

Barnett, T., J. C. Adam. and D. Lettenmaier, 2005: Potential impacts of a warming climate on water availability in snow-dominated regions, Nature, 438, 303-309.

Barnett T. P., D. W. Pierce, H. G. Hidalgo, C. Bonfils, B. D. Santer, T. Das, G. Bala, A. W. Wood, T. Nozawa, A. A. Mirin, D. R. Cayan, and M. D. Dettinger, 2008: 
Human-induced changes in the hydrology of the western United States, Science, 319, 1080-1083.

Bates, B. C., Z.W. Kundzewics, S. Wu, and J. P. Palutikof, 2008: Climate Change and Water. Technical Paper of the Intergovernmental Panel on Climate Change. IPCC Secretariat, Geneva, Switzerland, 210 pp.

Emori, S., 2006: Prediction of climate change and reliability of high resolution climate model, Global Environment, vol. 11, ppt. 3-10. (in Japanese)

Goswami, B. N., V. Venugopal, D. Sengupta, M. S. Madhusoodanan, and Prince K. Xavier, 2006: Increasing trend of extreme rain events over India in a warming environment, Science, 314, 1442-1445, doi:10.1126/ science.1132027.

Hirabayashi, Y., S. Kanae, S. Emori, T. Oki, and M. Kimoto, 2008: Global projections of changing risks of floods and droughts in a changing climate, Hydrological Sciences, 53, 754-772.

Hoshi, K., 1998: Probabilistic evaluation of flood peaks, Monthly Report of the Civil Engineering Research Institute of Hokkaido, 539, 34-37, (in Japanese).

Huffman, G. J., R. F. Alder, D. T. Bolvin, and G. Gu, 2009: Improving the global precipitation record: GPCP Version 2.1, Geophys. Res. Lett., 36, L17808, doi:10.1029/2009GL040000

Huntington, T. G., 2006: Evidence for intensification of the global water cycle: Review and synthesis, J. Hydrol., 319.

Intergovernmental Panel on Climate Change (IPCC) Fourth Assessment Report, 2007: Climate Change 2007: The Physical Science Basis. Contribution of Working Group 1 to the Fourth Assessment Report of the Intergovernmental Panel on Climate Change (Cambridge Univ. Press, Cambridge, UK, and New York)

Intergovernmental Panel on Climate Change (IPCC) Fourth Assessment Report, 2007: Climate Change 2007: Impacts, Adaptation, and Vulnerability. Contribution of Working Group 2 to the Fourth Assessment Report of the Intergovernmental Panel on Climate Change (Cambridge Univ. Press, Cambridge, UK, and New York)

Jung, M., M. Reichstein, P. Ciais, S. I. Seneviratne, J. Sheffield, M. L. Goulden, G. Bonan, A. Cescatti, J. Chen, R. de Jeu, A. J. Dolman, W. Eugster, D. Gerten, D. Gianelle, N. Gobron, J. Heinke, J. Kimball, B. E. Law, L. Montagnani, Q. Mu, B. Mueller, K. Oleson, D. Papale, A. D. Richardson, O. Roupsard, S. Running, E. Tomelleri, N. Viovy, U. Weber, C. Williams, E. Wood, S. Zaehle, and K. Zhang, 2010: Recent decline in the global land evapotranspiration trend due to limited moisture supply, Nature, 467, 951-954, doi:10.1038/ nature09396.

Kundzewicz, Z. W., L. J. Mata, N. W. Arnell, P. Doll, P. Kabat, B. Jimenez, K. A. Miller, T. Oki, Z. Sen, and I. A. Shiklomanov, 2008: The implications of projected climate change for freshwater resources and their man- agement. Hydrological Sciences, 53, 3-10.

Labat, D., Y. Godderis, J. L. Probst, and J. L. Guyot, 2004: Evidence for global runoff increase related to climate warming. Adv. Water Resour, 27, 631-642.

Matthews, D., 2006: The water cycle freshens up, Nature, 439, 793-794.

Milly, P. C. D., K. A. Dunne, and A. V. Vecchia, 2005: Global pattern of trends in streamflow and water availability in a changing climate, Nature, 438, 347-350, doi:10.1038/nature04312.

Min, S. -H., X. Zhang, F. W. Zwiers, and G. C. Hegerl, 2011: Human contribution to more-intense precipitation extremes, Nature, doi:10.1038/nature09763.

Ngo-Duc, T., T. Oki, and S. Kanae, 2007: A variable streamflow velocity method for global river routing model: model description and preliminary results, Hydrology and Earth System Sciences Discussions, 4, 4389-4414

Nitta, T., K. Yoshimura, S. Kanae, and T. Oki, 2010: Change of flood risk under climate change based on discharge probability index in Japan, Conference on Hydraulic Engineering, vol. 54.

Nohara, D., A. Kitoh, M. Hosaka, and T. Oki, 2006: Impact of Climate Change on River Discharge Projected by Multimodel Ensemble, Journal of Hydrometeorology, 7, 1076-1089.

Oki, T., and Y. C. Sud, 1998: Design of Total Runoff Integrating Pathways (TRIP) - A Global River Channel Network, Earth Interactions, vol. 2.

Oki, T., and S. Kanae, 2006: Global hydrological cycles and world water resources, Science, 313(5790), 10681072.

Special Report on Managing the Risks of Extreme Events and Disasters to Advance Climate Change Adaptation (SREX): 2011.

Thai Government, Emergency Operation Center for flood, Storm and Landslide, 2011, Flood Situation Reports

Trenberth, K. E., 1998: Atmospheric moisture residence times and cycling: Implications for rainfall rates and climate change, Clim. Change, 39, 667-694, doi:10.1023/A:1005319109110.

Trenberth, K. E., A. Dai, R. M. Rasmussen, and D. B. Parsons, 2003: The changing character of precipitation, Bull. Amer. Meteor. Soc., 84, 1205-1217, doi:10.1175/ BAMS-84-9-1205.

Watanabe, M., T. Suzuki, R. Oishi, Y. Komuro, S. Watanabe, S. Emori, T. Takemura, M. Chikira, T.Ogura, M. Sekiguchi, K. Takata, D. Yamazaki, T. Yokohata, T. Nozawa, H. Hasumi, H. Tatebe, and M. Kimoto, 2010: Improved climate simulation by MIROC5: Mean states, variability, and climate sensitivity, J. Climate, 23, 6312-6335.

The World Bank, 2011, The World Bank Supports Thailand's Post-Floods Recovery Effort

Yamazaki, D., T. Oki, and S. Kanae, 2009: Deriving a global river network map at flexible resolutions from a fine-resolution flow direction map with explicit rep- 
resentation of topographical characteristics in subgrid scale, Hydrology and Earth System Sciences, 13, 2241-2251.

Yoshimura, K., T. Sakimura, T. Oki, S. Kanae, and S. Seto,
2008: Toward flood risk prediction: a statistical approach using a 29-year river discharge simulation over Japan, Hydrological Research Letters, 2, 22-26. 